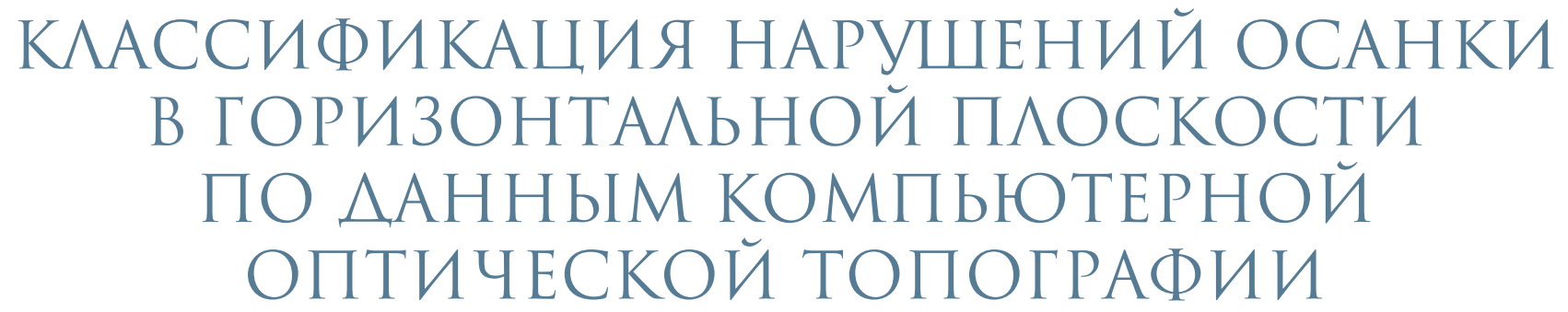

B.Н. Сарнадскии́

ООО «Медицинские топографические системы «МЕТОС», Новосибирск

\begin{abstract}
Цель исследования. Анализ уточненной классификации нарушений осанки в горизонтальной плоскости, учитывающей разные варианты нарушений, и исследование с использованием этой классификации структуры ротационных нарушений осанки.

Материал и методы. По результатам скриниг-обследования детеи́ и подростков методом компьютерной оптической топографии (KOMOT) в шести городах России собрана клиническая база более 33000 пациентов с приблизительно равномерным распределением по возрасту, от 4,5 до 17,5 лет (средний возраст $11,17 \pm 3,3$ года).

Результаты. Разработана уточненная классификация нарушений осанки в горизонтальной плоскости по данным метода КОМОТ на основе количественных топографических критериев. Классификация дифференцирует ротационные нарушения осанки по тяжести на слабовыраженные, умеренные и выраженные отклонения и разделяет умеренные и выраженные отклонения с учетом соотношения поворота плечевого пояса и таза на три варианта ротационных нарушений.

Заключение. С использованием предложенной классификации проведено исследование, которое показало существенно более слабое различие по половому признаку структуры ротационных нарушений осанки у детеи́ и подростков по сравнению с нарушениями в сагиттальной плоскости.

Ключевые слова: компьютерная оптическая топография, классификация нарушений осанки в горизонтальной плоскости.

\section{CLASSIFICATION OF HORIZONTAL PLANE POSTURAL DISORDERS AS EVIDENCED BY COMPUTER OPTICAL TOPOGRAPHY V.N. Sarnadsky}

Objective. To analyze the exacted classification of horizontal plane postural disorders, accounting for different variants of disorders, and to study the structure of rotational postural disorders using this classification.

Material and Methods. Results of the computer optical topography (COMOT) screening of children and adolescents in six cities formed a clinical database including information from more than 33,000 patients approximately evenly distributed through ages from 4.5 to 17.5 years (mean age $11.17 \pm 3.3$ years $)$.

Results. A new classification of horizontal plane postural disorders was elaborated based on the COMOT method findings using quantitative topographic criteria. This classification differentiates rotational postural disorders depending on their severity into mild, moderate and marked deviations, and divides moderate and marked deviations into three variants of rotational disorders according to the ratio of rotation of the shoulder girdle and pelvis.

Conclusion. The study performed using the proposed classification showed a significantly weaker gender difference in the structure of rotational postural disorders in children and adolescents compared with disorders in the sagittal plane.

Key Words: computer optical topography, classification, postural disorders, horizontal plane.

Hir. Pozvonoc. 2011; (4):40-46.

Оценку осанки до недавних пор проводили только в двух плоскостях: в сагиттальной исследовали состояние физиологических изгибов, а во фронтальной оценивали наличие бокового отклонения позвоночного столба и асимметрию расположения отдельных частей тела. При этом под пра-

вильной (физиологичной) осанкой большинством специалистов понимается следующее:

- прямое положение головы и симметричность шейно-плечевых линий;

- одинаковый уровень лопаток и отсутствие их чрезмерного расхождения;

- одинаковый уровень расположения подмышечных складок;

- симметричность треугольников талии, образованных внутренним краем верхних конечностей и боковой поверхностью туловища;

- нормальная величина физиологических изгибов позвоночника;
\end{abstract}


- прямое положение линии остистых отростков.

Состояние ориентации туловища и его частей в горизонтальной плоскости долгое время оставалось без внимания со стороны ортопедов, что вполне объяснимо, так как на практике использовали только визуальную оценку с применением нехитрых приспособлений и инструментов типа отвеса и линейки. По словам известного отечественного специалиста в вопросах осанки А.А. Потапчук [7], последняя попытка классификации нарушений осанки относится к началу 1960-х гг. За прошедший период, несмотря на то что значительно изменилось представление о состояниях, сопровождающих нарушения осанки, и механизмах их формирования, существенных изменений в классификации нарушений осанки не произошло (нарушения осанки оценивают во фронтальной и сагиттальной плоскостях).

Ситуация изменилась благодаря появлению в России в 1994 г. нового метода инструментальной диагностики - компьютерной оптической топографии (КОМОТ) и системы ТОДП, реализующей этот бесконтактный метод обследования пациентов, что обеспечило возможность комплексной количественной оценки нарушений осанки сразу в трех плоскостях: фронтальной, сагиттальной и горизонтальной [3, 11]. В 2004 г. в рамках реализации программы правительства Москвы «Диагностика и коррекция нарушений осанки у школьников» (2003-2005 гг.) автором совместно с московскими врачамиортопедами, участниками программы, для интерпретации результатов скрининг-обследования на системе ТОДП был разработан формализованный топографический диагноз для оценки состояния осанки в трех плоскостях [12]. С учетом последующего его применения на практике и анализа накопленного по результатам топографического скрининга статистического материала в 2011 г. разработана уточненная трехплоскостная классификация нарушений осанки и деформа- ции позвоночника по данным КОМОТ. Классификации нарушений осанки в сагиттальной плоскости была посвящена предшествующая статья [10].

В основе этой трехплоскостной классификации лежит понятие гармоничной осанки, критериями которой является следующее:

- оптимальная статика туловища во фронтальной и сагиттальной плоскостях;

- отсутствие скручивания туловища (поворота плечевого пояса относительно таза) в горизонтальной плоскости;

- симметрия туловища относительно срединной линии;

- отсутствие боковых искривлений позвоночника;

- сбалансированность физиологических изгибов и их анатомически правильное положение;

- соответствие основных параметров формы дорсальной поверхности туловища среднестатистическим значениям, полученным по результатам топографического скрининга большого массива пациентов в во3расте от 5 до 17 лет.

По существу, понятие гармоничной осанки вполне согласуется с общепринятым понятием правильной осанки, в дополнение к критериям которой введено следующее: отсутствие скручивания туловища в горизонтальной плоскости, анатомически правильное положение физиологических изгибов вдоль оси позвоночника и оптимальная статика (баланс) туловища в сагиттальной плоскости, а также соответствие основных топографических параметров дорсальной поверхности туловища среднестатистическим. Главным же отличием предлагаемой классификации от существовавших ранее, помимо оценки состояния осанки в горизонтальной плоскости, является использование количественных критериев. Выраженность отклонений от гармоничного состояния осанки оценивается на основе б-нормированных топографических параметров: $\mathrm{P}_{\sigma}=\left(\mathrm{P}-\mathrm{P}_{\mathrm{H}}\right) / \sigma_{\mathrm{p}}$, где $\mathrm{P}-$ значение топографического параметpa; $\mathrm{P}_{\mathrm{H}}$ - значение нормы для данно- го параметра (среднестатистическое значение параметра для детей и подростков); $\sigma_{\mathrm{p}}-$ среднеквадратическое отклонение.

В формализованном диагнозе для горизонтальной плоскости [12] основным критерием нарушений осанки служил топографический параметр GT, который рассчитывается как разность угла поворота в горизонтальной плоскости плечевого пояса $(\mathrm{GH})$ и таза $(\mathrm{GP})$ и описывает разворот плечевого пояса относительно таза, то есть скрученность туловища или баланс туловища в горизонтальной плоскости. На рис. 1 приведена гистограмма для параметра GT, которая достаточно точно соответствует нормальному закону распределения (коэффициент асимметрии распределения составил -0,01).

Для горизонтальной плоскости в формализованном топографическом диагнозе, как и для других плоскостей, состояние осанки дифференцировали по степени тяжести на зеленую, желтую и красную зоны. Зеленая зона включает норму (3Н), для которой $\sigma$ нормированый параметр GT лежит в пределах от $-2 / 3$ до 2/3, и субнорму (3C), для которой $\mathrm{GT}_{\sigma}$ в пределах от $-2 / 3$ до -1 и от $2 / 3$ до 1 . К желтой зоне относится состояние «нарушение осанки - ротированная осанка» (НО$\mathrm{PO})$, для которого GT ${ }_{\sigma}$ в пределах от -1 до -2 и от 1 до 2; к красной - «деформация позвоночника - ротированный позвоночник» (ДП-РП), для которого

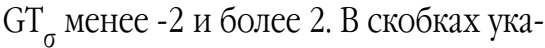
заны краткие обозначения состояния осанки в горизонтальной плоскости, используемые в старом формализованном диагнозе системы ТОДП.

Предложенный подход для анализа состояния осанки пациентов в горизонтальной плоскости на практике доказал свою состоятельность и нашел понимание со стороны отечественных ортопедов. Подтверждением этого могут служить работы авторов, исследующих с помощью топографии нарушения осанки в горизонтальной плоскости $[1,2,4-6,8,9,13]$.

Однако анализ результатов топографического скрининга показал, 
что у пациентов встречается три варианта разворота плечевого пояса относительно таза: разнонаправленный поворот плечевого пояса и таза (скручивание туловища), ротационное нарушение с преобладанием поворота таза и ротационное нарушение с преобладанием поворота плечевого пояса. Это потребовало дальнейшей доработки классификации нарушений осанки в горизонтальной плоскости по данным КOMOT.

Цель исследования - анализ уточненной классификации нарушений осанки в горизонтальной плоскости, учитывающей разные варианты нарушений, и исследование с использованием этой классификации структуры ротационных нарушений осанки.

\section{Материал и методы}

В рамках настоящего исследования использовали клиническую базу скрининг-обследований «МЕТОС» (КБСОМ), которая специально создана автором для научных исследований. В КБСОМ вошли результаты скрининг-обследований детского населения на системах ТОДП второго поколения (с фиксированной оптической системой) в шести городах России: Абакане, Новосибирске, Омске, Перми, Тольятти и Тюмени. База включает более 33000. пациентов (около 5500 человек на каждый город с примерно равномерным распределением по полу и возрасту от 4,5 до 17,5 лет; общее число мальчиков - 16576, средний возраст 11,11 $\pm 3,26$; общее число девочек - 16948, средний возраст $11,22 \pm 3,33)$. Из исходных баз удалены все некорректные снимки пациентов, а некорректно обработанные снимки переобработаны автором.

\section{Результаты и их обсуждение}

Проведенные исследования позволили создать уточненную классификацию нарушений осанки в горизонтальной плоскости по данным КОМОТ. Классификация содержит 8 состояний осанки в зависимости от тяжести и варианта ротационных нарушений. Зеленая зона не дифференцируется по вариантам нарушений и включает в себя гармоничную осанку и субнорму (осанку с незначительными ротационными нарушениями). Желтая и красная зоны дифференцируются на три варианта ротационных нарушений каждая. В табл. приведены краткое описание этих 8 состояний осанки в горизонтальной плоскости и соответствующие им количественные топографические критерии.

Зеленая зона в новой классификации осталась без изменений и содержит норму (3Н - гармоничное состояние) и субнорму (3С - слабовыраженное скручивание туловища). В желтой зоне вместо одного состояния «нарушение осанки - ротированная осанка» (НО-РО - умеренное скручивание туловища) вводятся три состояния: «нарушение осанки - скрученное туловище» (НО-СТл - умеренно скрученное туловище), «нарушение осанки - ротированный таз» (НО-РТз - умеренно ротированный таз) и «нарушение осанки - ротированный плечевой пояс» (НО-РПл - умеренно ротированный плечевой пояс). В красной зоне вместо одного состояния «деформация позвоночника - ротированный позвоночник» (ДП-РП) вводятся три состояния: «деформированный позвоночник - гиперскрученное туловище» (ДП-ГТл - выраженно скрученное туловище), «деформированный позвоночник - гиперротированный таз» (ДП-ГТз - выраженно ротированный таз) и «деформированный

\begin{tabular}{|c|c|c|c|c|}
\hline № & Обозначение & Наименование & Краткое описание & Топографические критерии \\
\hline 1 & $3 \mathrm{H}$ & 3доров - норма & Осанка, близкая к гармоничной & $\left|\mathrm{GT}_{\sigma}\right|<2 / 3$ \\
\hline 2 & $3 \mathrm{C}$ & Субнорма & $\begin{array}{l}\text { Слабовыраженные ротационные нарушения } \\
\text { осанки в пределах зеленой зоны }\end{array}$ & $2 / 3 \leq\left|\mathrm{GT}_{\sigma}\right|<1$ \\
\hline 3 & НО-СТл & Скрученное туловище & $\begin{array}{l}\text { Разнонаправленный разворот плечевого пояса } \\
\text { и таза в пределах желтой зоны }\end{array}$ & $\begin{array}{l}1 \leq\left|G_{\sigma}\right|<2 \& \\
\& \mid \text { GH-Gcor }\left|<2^{\star}\right| \text { GP-Gcor } \mid \& \\
\& \mid \text { GP-Gcor }\left|<2^{*}\right| \text { GH-Gcor } \mid\end{array}$ \\
\hline 4 & $\mathrm{HO}-\mathrm{PT} 3$ & Ротированный таз & $\begin{array}{l}\text { Разворот таза по или против часовой стрелки } \\
\text { в пределах желтой зоны }\end{array}$ & $\begin{array}{l}1 \leq\left|\mathrm{GT}_{\sigma}\right|<2 \& \\
\& \mid \text { GP-Gcor }\left|\geq 2^{*}\right| \text { GH-Gcor } \mid\end{array}$ \\
\hline 5 & НО-РПл & Ротированный плечевой пояс & $\begin{array}{l}\text { Разворот плечевого пояса по или против часовой } \\
\text { стрелки в пределах желтой зоны }\end{array}$ & $\begin{array}{l}1 \leq\left|\mathrm{GT}_{\sigma}\right|<2 \& \\
\& \mid \mathrm{GH}-\text {-Gcor }\left|\geq 2^{*}\right| \text { GP-Gcor } \mid\end{array}$ \\
\hline 6 & АП-ГТл & Гиперскрученное туловище & $\begin{array}{l}\text { Разнонаправленный разворот плечевого пояса } \\
\text { и таза в пределах краснои́ зоны }\end{array}$ & $\begin{array}{l}2 \leq\left|\mathrm{GT}_{\sigma}\right| \& \\
\& \mid \text { GH-Gcor }\left|<2^{*}\right| \text { GH-Gcor } \mid \& \\
\& \mid \text { GP-Gcor }\left|<2^{*}\right| \text { GH-Gcor } \mid\end{array}$ \\
\hline 7 & АП-ГТз & Гиперротированный таз & $\begin{array}{l}\text { Разворот таза по или против часовой стрелки } \\
\text { в пределах красной зоны }\end{array}$ & $\begin{array}{l}2 \leq\left|\mathrm{GT}{ }_{\sigma}\right| \& \\
\& \mid \text { GP-Gcor }|\geq 2 *| \text { GH-Gcor } \mid\end{array}$ \\
\hline 8 & АП-ГПл & Гиперротированныи́ плечевой пояс & $\begin{array}{l}\text { Разворот плечевого пояса по или против часовой } \\
\text { стрелки в пределах красной зоны }\end{array}$ & $\begin{array}{l}2 \leq\left|\mathrm{GT}_{\sigma}\right| \& \\
\& \mid \mathrm{GH}-\text { Gcor }|\geq 2 *| \text { GP-Gcor } \mid\end{array}$ \\
\hline
\end{tabular}


позвоночник - гиперротированный плечевой пояс» (ДП-ГПл - выраженно ротированный плечевой пояс).

В предлагаемой классификации, как и в старой, основным параметром для разделения ротационных нарушений осанки по выраженности служит $\mathrm{GT}_{\sigma}-\sigma$-нормированный параметр, оценивающий разворот плечевого пояса относительно таза. Полученное по базе КБСОМ значение $\sigma$ для параметра GT составило 3,15 (см. среднее квадратическое отклонение на рис. 1). Для разделения ротационных нарушений в желтой и красной зонах по варианту нарушений используется соотношение между параметрами GH и GP: если GH болыше GP в два раза и более, то нару- шения относятся к ротированному плечевому поясу; если GP болыше GH в два раза и более, то нарушения относятся к ротированному тазу; если GP и $\mathrm{GH}$ отличаются менее, чем в два раза, а GT болыше 1 или меныше -1, то есть имеется взаимный поворот плечевого пояса относительно таза, то нарушения относятся к скрученному туловищу.

Следует отметить, что при обследовании на системе ТОДП используется автокоррекция поворота туловища в горизонтальной плоскости, посредством которой туловище разворачивается так, чтобы его дорсальная поверхность имела минимальный поворот относительно фронтальной плоскости. Коррекция поворота производится

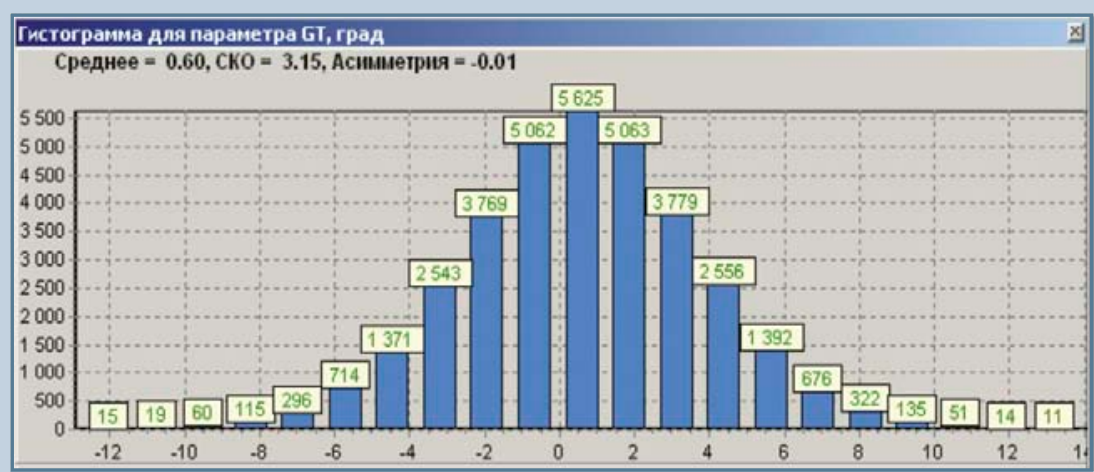

Рис. 1

Гистограмма топографического параметра GT, описывающего разворот плечевого пояса относительно таза

на угол, задаваемый параметром $G_{\text {сог }}$. За счет этой коррекции значения параметров GH и GP становятся относительными (к их значениям добавляется $\mathrm{G}_{\mathrm{cor}}$ ). Для правильной классификации требуются абсолютные значения, поэтому в критериях табл. из параметров GH и GP вычитается значение $G_{\text {cor. }}$ Это позволяет получать абсолютные углы разворота таза и плечевого пояса относительно линии, соединяющей пятки левой и правой стоп. Условия корректной съемки пациента на системе ТОДП требуют, чтобы стопы пациента на установочном месте были выровнены по пяткам постановкой их с упором в специальную планку. При соблюдении данного условия линия, соединяющая пятки пациента, параллельна фронтальной плоскости, поэтому можно корректно оценивать ориентацию туловища относительно стоп. В противном случае (при отсутствии корректной установки стоп) диагностика по виду ротационных нарушений осанки может быть недостоверной.

В топографических критериях, представленных в табл., ряд топографических параметров используют без учета знака, что обозначается, например, как |GT ${ }_{\sigma} \mid$. Сами критерии задаются с помощью отдельных условий или условий, объединяемых посредством логического «И» (символ «\&»), что означает одновременное выполнение этих условий.

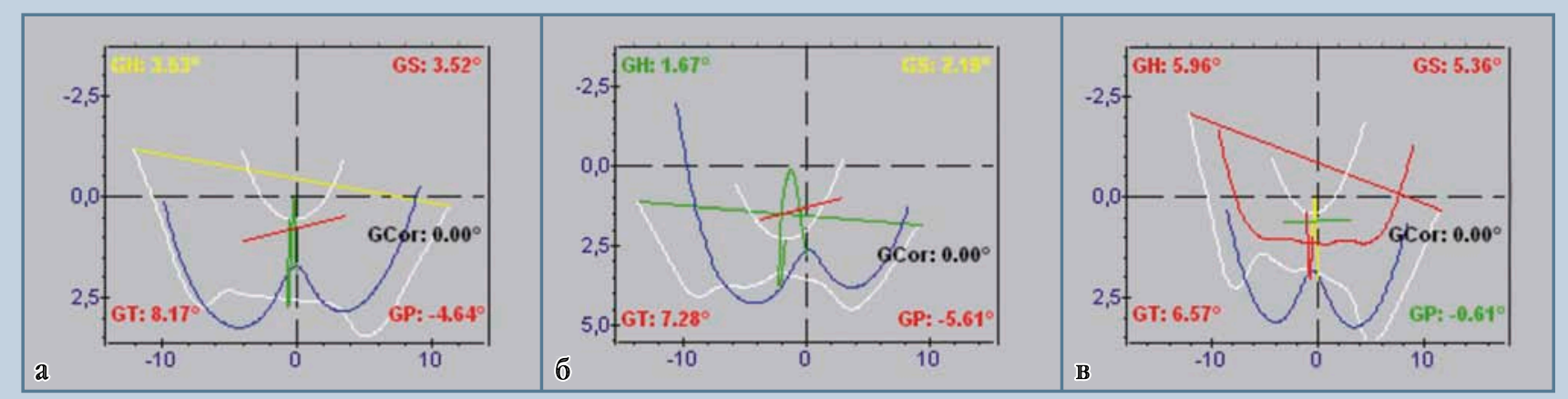

Рис. 2

Примеры выраженных ротационных нарушений осанки в горизонтальной плоскости: а - гиперскрученное туловище; б - гиперротированный таз; в - гиперротированный плечевой пояс 
На рис. 2 приведены варианты ротационных нарушений в красной зоне. Для наглядности восприятия при обработке этих примеров отменена автокоррекция поворота $\left(G_{\text {cor }}=0\right)$.

На рис. 3 представлена структура нарушений осанки в горизонтальной плоскости, полученная по базе КБСОМ, отдельно для мужского и женского пола. Зеленая зона у мальчиков составляет $69,78 \%$, что на $1,2 \%$ больше, чем у девочек (68,56\%). Желтая зона у мальчиков - 25,94\%, у девочек - 26,13\%, то есть практически одинакова. Красная зона у мальчиков - 4,28\%, что на $1,03 \%$ меньше, чем у девочек (5,31\%).

Структура по вариантам ротационных нарушений в желтой зоне у мальчиков и девочек практически совпадает: первое место по встреча-

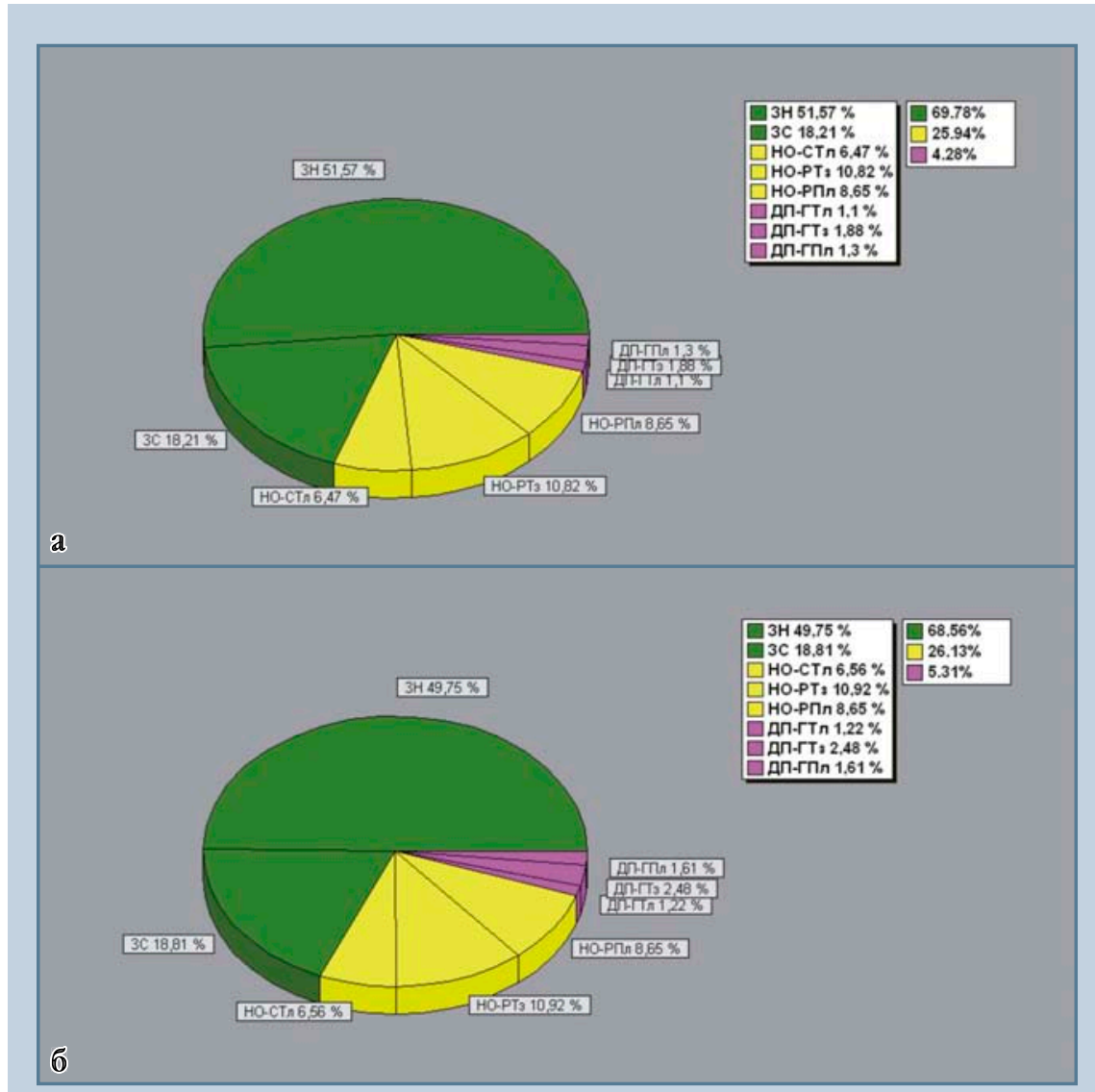

Рис. 3

Структура ротационных нарушений осанки в горизонтальной плоскости у пациентов с 4,5 до 17,5 лет: а - мужского пола; 6 - женского пола

емости занимает ротированный таз $(10,82 \%$ у мальчиков и $10,92 \%$ у девочек); второе - ротированный плечевой пояс $(8,65 \%$ у мальчиков и $8,65 \%$ у девочек); третье - скрученное туловище $(6,47 \%$ у мальчиков и $6,56 \%$ у девочек). В красной зоне структура по вариантам ротационных нарушений у мальчиков и у девочек совпадает качественно, но несколько отличается количественно (для всех вариантов у девочек увеличен процент встречаемости). Как и в желтой зоне, первое место по встречаемости занимает ротированный таз $(1,88 \%$ у мальчиков и $2,48 \%$ у девочек), второе - ротированный плечевой пояс (1,30\% у мальчиков и 1,61 \% у девочек), третье - скрученное туловище $(1,10 \%$ у мальчиков и 1,22 \% у девочек). 


\section{Литература}

1. Батршин И.Т. Характеристика и роль ротационных нарушений позвоночника // Экспертиза отдаленных результатов лечения травм и ортопедических заболеваний у детей: Тез. докл. конф. М., 2007. C. 29-30.

2. Величко М.Н., Колмыков В.В., Новохатский А.П.

и др. Первый опыт скрининг-обследования школьников г. Красноярска методом компьютерной оптической топографии // Диагностика, профилактика и коррекция нарушений опорно-двигательного аппарата у детей и подростков: Тез. докл. II Всерос. науч.-практ. конф. с междунар. участием. M., 2008. C. 25-26.

3. Евразийский пат. № 000111. Способ компьютерной оптической топографии тела человека и устройство для его осуществления / Сарнадский В.Н., Садовой М.А., Фомичев Н.Г;; заявл.26.08.1996; опубл. 27.08.1998, Бюл. № 4.

4. Колчин Д.В., Шатохин В.Д., Вишнякова О.Н. Место оптической компьютерной топографии в ранней скрининговой диагностике ортопедических заболеваний у детей г. Тольятти // Травматология и ортопедия XXI века: Тез. докл. конф. Самара, 2006. С. 915-916.

5. Любченко В.Ю. Диагностика и коррекция нарушений осанки. Тольятти, 2004

6. Мавлиева Г.М., Шайхелисламов Р.Р., Степанов А.А. и др. Компьютерное топографическое скрининг-обследование школьников Республики Татарстан // Диагностика, профилактика и коррекция нарушений опорно-двигательного аппарата у детей и подростков: Тез. докл. II Всерос. науч.-практ. конф. с междунар. участием. М., 2008. С. 97-98.

7. Потапчук А.А., Дидур М.Д. Осанка и физическое развитие детей. Программы диагностики и коррекции нарушений. СПб., 2001.

8. Псянчин Т.С., Батршин И.Т., Юнусов Д.И. и др. Индексы нарушений и ориентации туловища в горизонтальной плоскости при деформациях позвоночника // Актуальные вопросы экспериментальной медицины и биологии: Тез. докл. II съезда травматологов-ортопедов Уральского федерального округа. Курган, 2008. С. 219-220.

9. Садовая Т.Н. Скрининг, мониторинг и организация специализированной ортопедической помощи детям с деформациями позвоночника: Дис. ... д-ра мед. наук. СПб., 2010.

10. Сарнадский В.Н. Классификация нарушений осанки в сагиттальной плоскости по данным ком- пьютерной оптической топографии // Хирургия позвоночника. 2011. № 3. С. 46-55.

11. Сарнадский В.Н., Фомичев Н.Г., Садовой М.А Мониторинг деформации позвоночника методом компьютерной оптической топографии. Новосибирск, 2001.

12. Сарнадский В.Н. Формализованный топографический диагноз по результатам скрининг-обследования пациентов в 4 позах // Диагностика, профилактика и коррекция нарушений опорнодвигательного аппарата у детей и подростков: Тез. докл II Всерос. науч.-практ. конф. с междунар. участием. М., 2008. С. 153-156.

13. Степкина М.А., Федотов В.К., Шкляренко А.П. Система диагностических и консервативных лечебных мероприятий при нарушениях осанки и деформациях позвоночника у детей и подростков. Омск, 2009.

\section{References}

1. Batrshin IT. [Characterization and role of rotational spinal disorders]. Expertize of Long-Term Results of Treatment of Injuries and orthopaedic Diseases in Children. Proceedings of the Conference, Moscow, 2007:29-30. In Russian.

2. Velichko MN, Kolmykov VV, Novohatsky AP, et al. [First experience of school screening in Krasnoyarsk city using Computer optical Topography]. Diagnosis, Prevention, and Correction of Locomotor System Disorders in Children and Adolescents. Proceedings of the 2nd All-Russian Scientific and practical Conference with international participation, Moscow, 2008:25-26. In Russian.

3. Sarnadsky VN, Sadovoy MA, Fomichev NG. [The Way to Perform Computer Optical Topography of Human Body and Device for its Implementation]. EA Patent 000111, filed 26.08.1996, publ. 27.08.1998. In Russian.

4. Kolchin DV, Shatohin VD, Vishnyakova ON. [A place of computer optical topography in early screening diagnosis of orthopaedic diseases in children from Togliatti town]. Traumatology and Orthopaedics of XXI Century. Proceedings of the Conference, Samara, 2006:915-916. In Russian.

5. Lyubchenko VYu. [Diagnosis and Correction of Postural Disorders]. Tol'yatti, 2004. In Russian.

6. Mavlieva GM, Shayhelislamov RR, Stepanov AA, et al. [Computer Topography Screening of Schoolchildren in the Republic of Tatarstan]. Diagnosis, Prevention, and Correction of Locomotor System Disorders in Children and Adolescents. Proceedings of the 2nd All-Russian Scientific and practical Conference with international participation, Moscow, 2008:97-98. In Russian.

7. Potapchuk AA, Didur MD. [Posture and Physical Development of Children. Programs of Disorder Diagnosis and Correction]. St. Petersburg, 2001. In Russian.

8. Psyanchin TS, Batrshin IT, Yunusov DI, et al. [Indices of disorders and trunk orientation in horizontal plane in patients with spinal deformities]. Topical Issues of Experimental Medicine and Biology. Proceedings of the 2nd Congress of Traumatologists and Orthopedists of the Ural Federal District, Kurgan, 2008:219-220. In Russian.

9. Sadovaya TN. [Screening, Monitoring, and Organization of Specialized Orthopaedic Care to Children with Spinal Deformities]. Doctor of Medicine Thesis. St. Petrsburg, 2010. In Russian.

10. Sarnadsky VN. [Classification of sagittal plane postural disorders based on optical topography data]. Hir Pozvonoc. 2011;(3):46-55. In Russian.

11. Sarnadsky VN, Fomichev HG, Sadovoy MA. [Monitoring of Spinal Deformities Using Computer Optical Topography]. Novosibirsk, 2001. In Russian.

12. Sarnadsky VN. [Formalized diagnosis based on results of screening examination of patients in 4 positions]. Diagnosis, Prevention, and Correction of Locomotor System Disorders in Children and Adolescents. Proceedings of the 2nd All-Russian Scientific and practical Conference with international participation, Moscow, 2008:153-156. In Russian.

13. Stepkina MA, Fedotov VK, Shklyarenko AP. [System of Diagnostic and Conservative Interventions for Posture Disorders and Spinal deformities in Children and Adolescents]. Omsk, 2009. In Russian.

Адрес для переписки:

Сарнадский Владимир Николаевич

630091, Новосибирск, ул. Крылова, 31 ,

оф. 54, OOO «METOC»,

metos.org@gmail.com

Статья поступила в редакцию 08.09.2011 

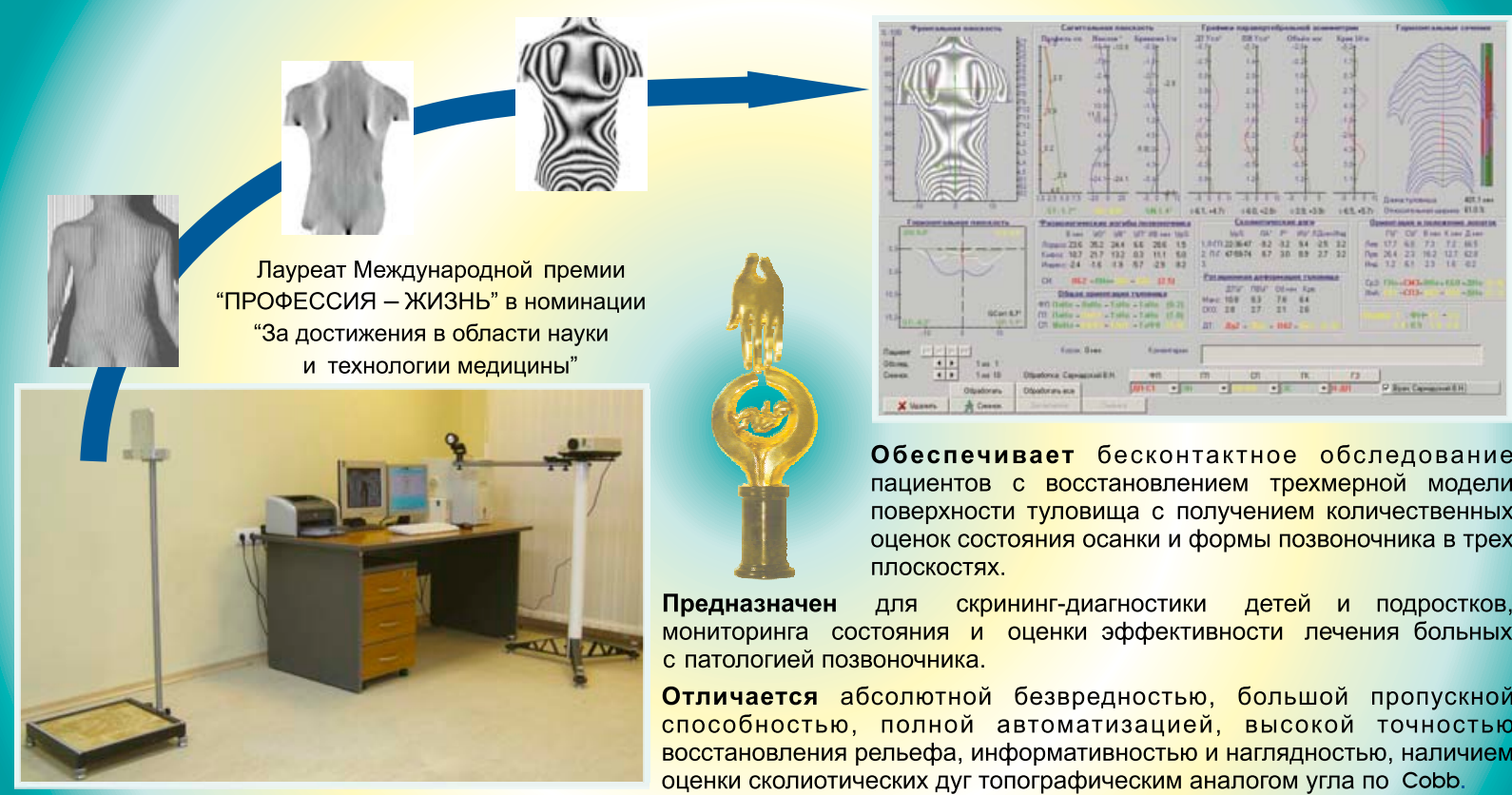

Предназначен для скрининг-диагностики детей и подростков, мониторинга состояния и оценки эффективности лечения больных с патологией позвоночника.

Отличается абсолютной безвредностью, большой пропускной способностью, полной автоматизацией, высокой точностью восстановления рельефа, информативностью и наглядностью, наличием оценки сколиотических дуг топографическим аналогом угла по Cobb.

16 лет клинической практики - более 210 установок ТОДП по России

Медицинское изделие ТОдП выпускается по лицензии Федеральной службы по надзору в сфере здравоохранения и социального развития № 99-03-000002. Сертификат соответствия № POCC RU.AЯ79.B02777.

630091, Новосибирск, ул. Крылова, 31, офис 54 ООО “МЕТОС” тел. (383) 325-41-50, http://www.metos.org, e-mail: metos.org@gmail.com

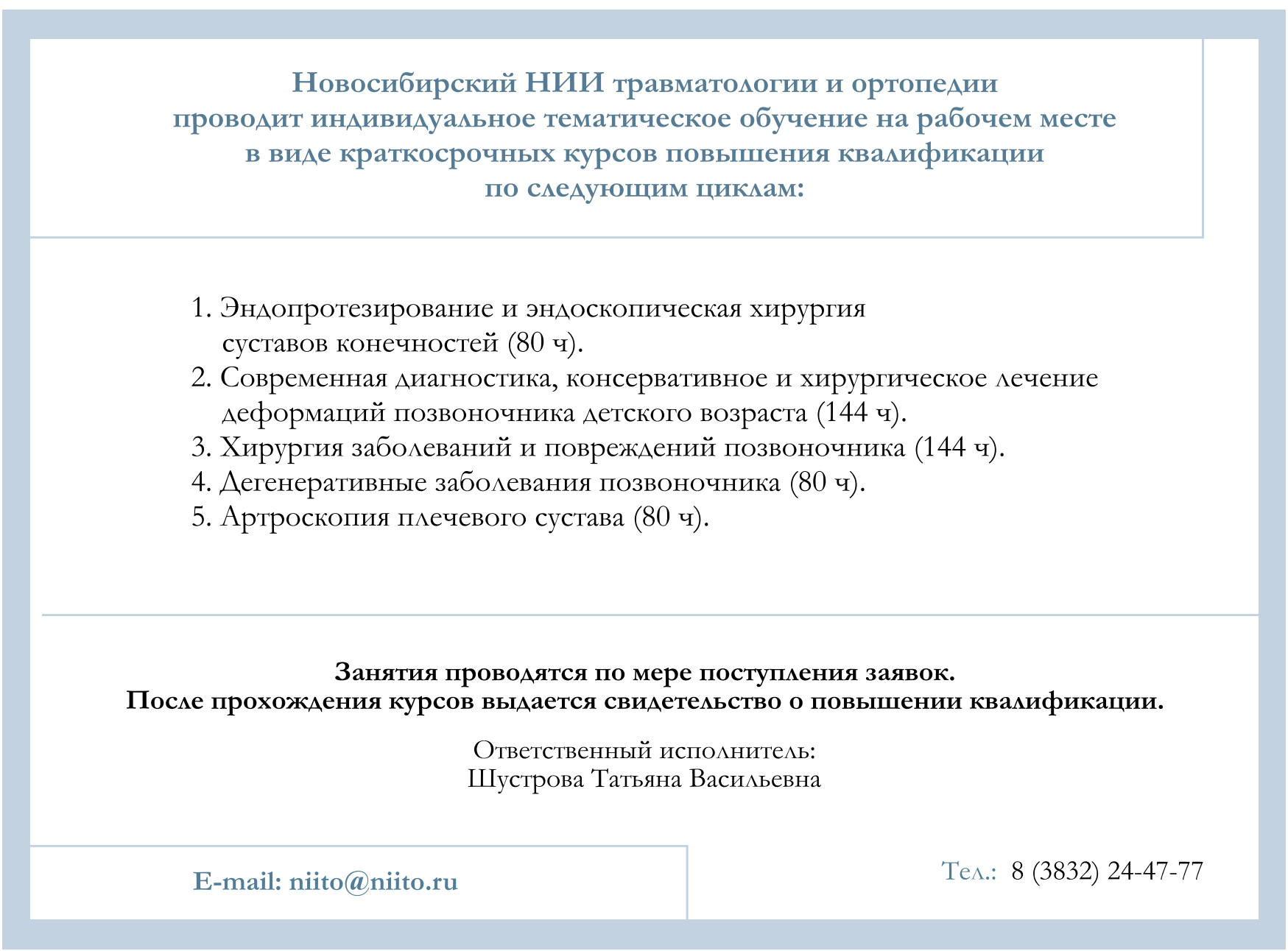

